\title{
Experimental investigation on vibration characteristics in thermosonics detection test
}

\author{
Gan Tian", a, Yin Li, Zhengwei Yang, Yong Jia, Anbo Ming, Wei Zhang \\ ${ }^{1}$ Xi'an Research Inst. Of Hi-Tech Hongqing, Xi'an.P.R.China \\ a tiangan_2012@163.com
}

\begin{abstract}
Keywords: thermosonics, nondestructive test, Vibration characteristics, Acoustic chaos Abstract. In order to investigate the vibration characteristics of the object in the nondestructive test using thermosonics, the device for measuring vibration micro-displacement was built based on the laser displacement sensor and the ultrasonic excitation equipment. On this basis, the vibration displacement of the object was measured under the condition of ultrasonic loading with the frequency of $40 \mathrm{kHz}$ and non-ultrasonic loading respectively. Then, the analysis on the frequency spectrum of vibration displacement obtained by the test was carried out. The obtained results show that there is fundamental frequency in the vibration spectrum of the object as well as the high-order harmonic frequencies, which confirms the existence of acoustic chaos in the thermosonics detection test. This work is of importance as it lays the foundation for the further investigation on the detection mechanism of thermosonics.
\end{abstract}

\section{Introduction}

Thermosonics is a new nondestructive testing technology [1-3], which uses ultrasonic wave as a heat source to cause object with surface damage to the surface damage to generate heat and utilizes the infrared camera to capture the thermographic images to observe the damage. This method is very suitable for detecting the joint damages such as surface/subsurface crack, delamination caused by impact etc..

Motivating the ultrasonic vibration of the object is the prerequisite for the nondestructive testing using thermosonics. Thus, the investigation on vibration characteristics attracts many attentions, especially the discovery of acoustic chaos phenomenon. In 2002, Xiaoyan Han etc. pointed out that the contact state between the ultrasonic gun and the specimen had an important influence on the generation of acoustic chaos, which did not depend on the existence of crack [4]. Moreover, they indicated that the presence of acoustic chaos could enhance the thermoraphic signal at the crack, and improved the detection capability of thermosonics [5, 6]. Afterwords, a series of numerical simulations on metal structures were carried out, which demonstrated the nonlinear vibration phenomena in the experiment through the analysis on the model vibration waveform and spectrum diagram of the metal structures [7]. In addition, I. Y. Solodov and B. A. Korshak pointed out that the generation of subharmonics was due to the existence of non-linear effect nearby the damage of object [8]. The authors of this paper [9, 10] investigated the vibration mode using finite element numerical simulation model and found the existence of the acoustic chaos in the thermosonics detection. But they did not perform the experiment to validate the simulation results.

Therefore, the device for measuring vibration micro-displacement was built based on the laser displacement sensor and the ultrasonic excitation equipment and the vibration displacement of the specimen was measured using laser displacement sensor. Then, the frequency spectrum of the object is obtained using FFT transform to investigate the acoustic chaos. 


\section{Experimental investigation}

In order to investigate the vibration characteristics in the thermosonics detection, the experimental device was built is shown in Fig. 1, including ultrasonic excitation source, laser sensor and computer control and processing system.

Ultrasonic excitation source is used to generate ultrasonic waves, with short pulses $(50 \sim 200 \mathrm{~ms})$ and low frequency $(20 \sim 40 \mathrm{kHz})$, on the surface of the specimen to motivate the vibration of the specimen The laser sensor is used to measure and record the vibration displacement of the specimen and transfer the displacement data to the computer. The computer is mainly used to deal with the displacement data.

In the experiment, the ultrasonic excitation equipment, with type of 2000LPt, is made by BRANSON with the maximum power of $4 \mathrm{~kW}$, excitation frequency of $40 \mathrm{kHz}$. The laser sensor with type of LK-G5000 series, is made by KEYENCE, with the high reproducibility of 0.005 um, ultra-high accuracy of $0.02 \%$, ultra-fast speed of $392 \mathrm{kHz}$.

In order to eliminate the influence of noise in the air, the test process is divided into the following two steps.

Step 1: under the condition of non-ultrasonic loading, the laser sensor is used to measure the vibration displacement of the specimen.

Step 2: under the condition of ultrasonic loading with the frequency of $40 \mathrm{kHz}$, the laser sensor is used to measure the vibration displacement of the specimen.

The vibration displacement data obtained by the above two steps were extracted respectively and the corresponding frequency spectrum was calculated by FFT is shown in Fig. 2. As is from in Fig. 2(a) and (b), despite the absence of ultrasonic loading, the higher harmonics $(140 \mathrm{kHz}, 200 \mathrm{kHz}$ and 270 $\mathrm{kHz}$ ) still occur, the reason is mainly due to such factors as air noise, airflow etc.. Fig.2 (c) and (d) show that in the theomosonics detection, there is not only the fundamental frequency $(40 \mathrm{kHz}) \mathrm{but}$ also the high-order harmonic frequencies $(80 \mathrm{kHz}, 320 \mathrm{kHz}, 360 \mathrm{kHz})$ in the vibration spectrum of the specimen. Compared with Fig.2 (b) and (d), it can be seen that the generation of higher harmonics in the specimen is due to the excitation of the ultrasonic loading, not the air noise or airflow, which proves the existence of acoustic chaos in the thermosonics detection, which is consistent with the numerical results in Refs. [10].

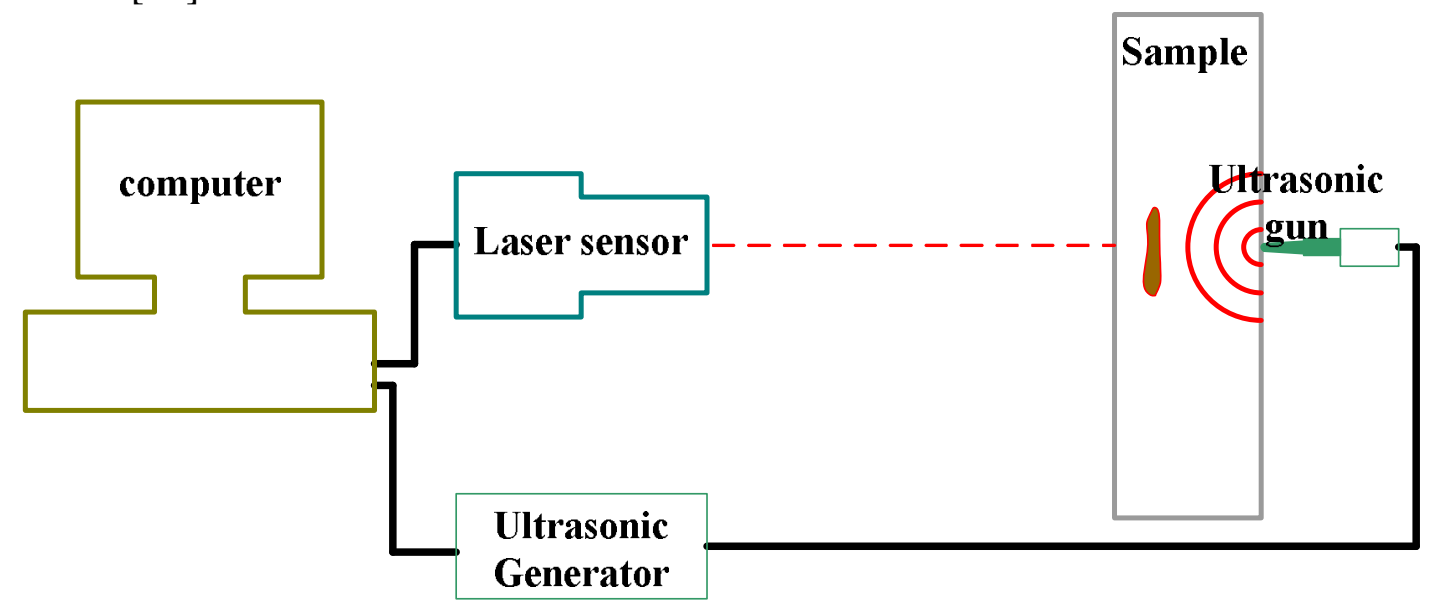

Fig. 1. Experimental equipment 

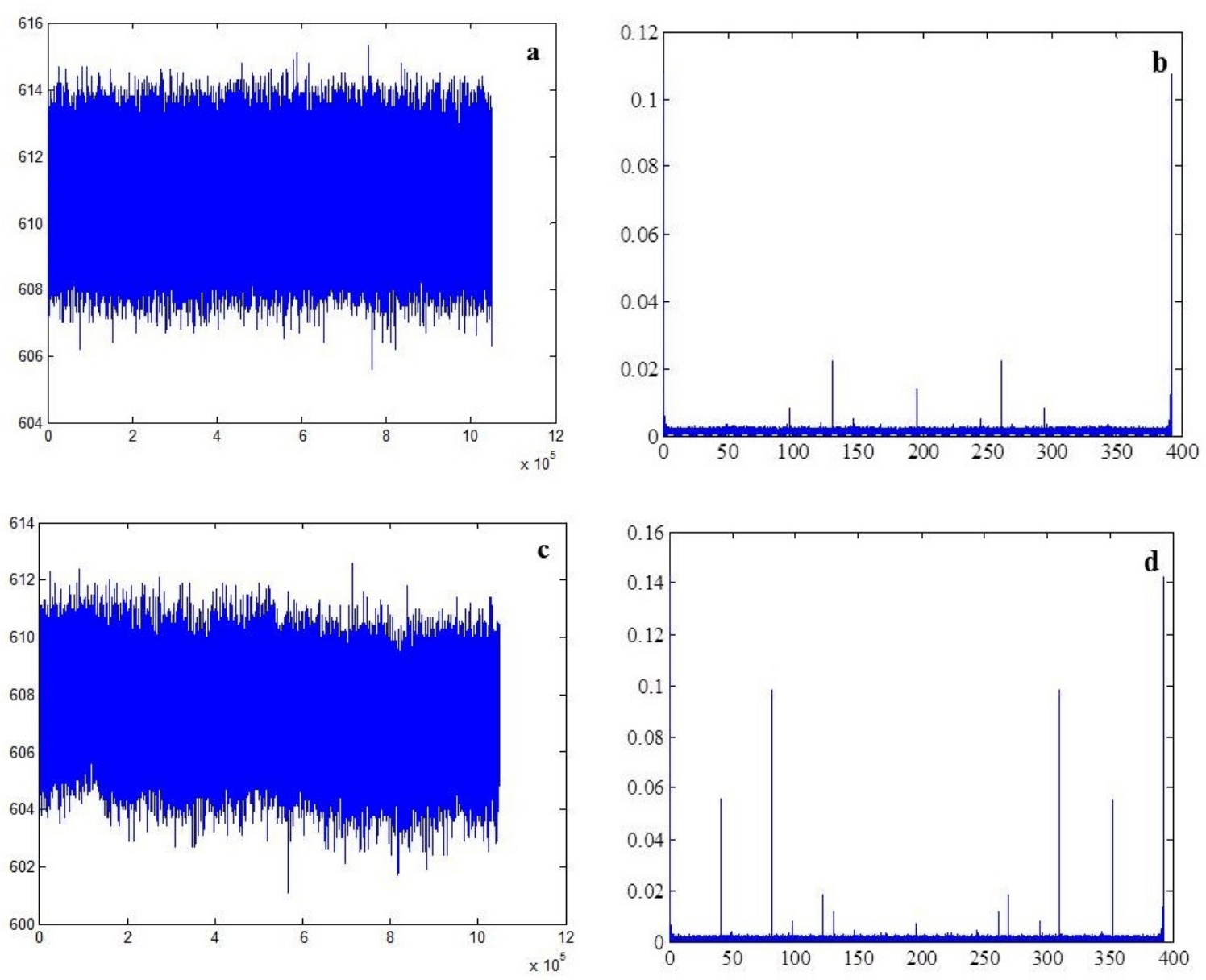

Fig. 2. Vibration displacement and corresponding frequency spectrum of the specimen. (a) Vibration displacement without ultrasonic loading. (b) Frequency spectrum without ultrasonic loading. (c) Vibration displacement under Ultrasonic Loading with the frequency of $40 \mathrm{kHz}$. (d) Frequency spectrum under ultrasonic loading with the frequency of $40 \mathrm{kHz}$.

\section{Conclusions}

In this work, the vibration displacement measurement of the specimen under two conditions (nonultrasonic loading and ultrasonic loading with the frequency of $40 \mathrm{kHz}$ ) was carried out. By analyzing the experimental data and frequency spectrum of vibration displacement of the specimen, the results show that there is not only the fundamental frequency but also the high-order harmonic frequencies in the vibration spectrum of the object, which confirms the existence of acoustic chaos in the thermosonics detection test.

\section{Acknowledgments}

This work was supported by the National Nature Science Foundation of China (Grant Nos. 51605481 and 51575516).

\section{References}

[1] Marco Morbidini, Peter Cawley, Tim Barden, et al. Prediction of the thermosonic signal from fatigue cracks in metals using vibration damping measurements [J]. Journal of Applied Physics, 2006, 100(10): 104905.1-13. 
[2] Vavilov V P. Dynamic thermal tomography: Recent improvements and applications [J]. NDT \& E International, 2015, 71: 23-32.

[3] R. D. Adams, P. Cawley, C. J. Pye, et al . A vibration technique for non-destructively assessing the integrity of structures [J] . Journal of Mechanical Engineering Science, 1978, 20(2): 93-100.

[4] Xiaoyan Han, Wei Li, and Zhi Zeng . Acoustic Chaos and Sonic Infrared Imaging [J] . Applied Physics Letters, 2002, 81(17): 3188-3190 .

[5] Xiaoyan Han, Zhi Zeng, Wei Li, et al . Importance of Acoustic Chaos in Sonic IR Imaging NDE [J] . Review of Quantitative Nondestructive Evaluation 23. AIP Conference Proceedings, 2004, 700: 496-500 .

[6] Xiaoyan Han, Zhi Zeng, Wei Li, et al . Acoustic Chaos for Enhanced Detectability of Cracks by Sonic Infrared Imaging [J] . Journal of Applied Physics, 2004, 95(7): 3792-3797 .

[7] Xiaoyan Han, Md. Sarwar Islam, G. Newaz, et al . Finite-Element Modeling of the Heating of Cracks in Sonic Infrared Imaging [J] . Journal of Applied Physics, 2006, 99(7): 074905.1-7 .

[8] Igor Yu Solodov, Boris A Korshak . Instability, chaos, and "memory" in acoustic-wave-crack interaction [J] . Physical review letters, 2002, 88(1): 014303.1-3.

[9] Tian Gan, Zhang Wei, Jin Guofeng, et al. Numerical simulation of multi-mode excitation of ultrasonic infrared thermal wave detection [J]. Journal of Jiangsu university, 2014, 35(2): 171-175. (in Chinese)

[10]Tian Gan, Yang Zhengwei, Zhu Jieteng, et al. Vibration characteristics and acoustic chaos analysis of ultrasonic infrared thermal wave test [J]. Infrared and Laser Engineering, 2016, 45(3): 0304003. (in Chinese) 